(Aus dem physiologischen Institut der Westfälischen Wilhelms-Universität Münster.)

\title{
Zur Physiologie und Pathologie der Säureabsonderung der Magenschleimhaut.
}

Von

\section{R. Rosemann.}

Die Säureabsonderung der Magenschleimhaut stellt das einfachste Beispiel einer Sekretion vor, das wir kennen. Denn während wir in anderen Sekreten stets eine ganze Anzahl verschiedener Bestandteile vor uns haben, enthält der Magensaft, wenn man von einer geringen Menge organischer Bestandteile absieht, fast nur Salzsäure und Chloride. Man sollte daher erwarten, daß der Vorgang und die Bedingungen der Sekretion gerade an diesem Beispiel am ehesten zu untersuchen sein müßten. Trotz aller Fortschritte, die uns auf diesem Gebiete besonders die Untersuchungen $\mathrm{Paw}$ lows ${ }^{1}$ ) gebracht haben, sind wir aber von einer klaren Einsicht in die Verhältnisse der Sekretion hier nicht weniger weit entfernt als bei andern Drüsen. Das gilt nicht nur rom physiologischen, sondern ebenso, wenn nicht in noch höherem Grade vom pathologischen Gebiet.

Durch die von $\mathrm{Pawlow}$ ausgearbeitete Untersuchungsmethode sind wir zum ersten Male in den Stand gesetzt worden, das reine Sekret der Magendrüsen in beliebiger Menge zur Untersuchung zu gewinnen. Nur dieses Sekret sollte als "Magensaft" bezeichnet werden. Es hat sich leider die Unsitte eingebürgert, auch das Filtrat des bei der klinischen Magenuntersuchung durch Ausheberung gewonnenen Mageninhaltes als ,Magensaft" zu bezeichnen. Dagegen muß man den schärfsten Einspruch erheben. Es handelt sich keineswegs hier etwa nur um eine schließlich ziemlich gleichgültige Frage der Bezeichnungsweise. Hätte man von Anfang an zwischen "Magensaft" und "Mageninhalt"s scharf unterschieden, so hätte nicht die irrtümliche Vorstellung aufkommen können, als ob man durch die Untersuchung des Mageninhaltes ohne weiteres auch etwas über die Zusammensetzung des Magensaftes erfahren könnte. Denn der Mageninhalt stellt ja natürlich eine Mischung aus den Bestandteilen der Probemahlzeit, verschlucktem Speichel und reinem Magensaft dar, sein Gehalt an den charakteristischen Bestandteilen des Magensaftes muß also jedenfalls niedriger sein als 
der Gehalt des reinen Magensaftes selbst. Will man auf Grund der Untersuchung des Mageninhalts die Zusammensetzung und Menge des abgesonderten Magensaftes durch Rechnung feststellen, so müßte dazu nicht nur die Zusammensetzung und Menge der Probemahlzeit und des bei ihrer Aufnahme mitverschluckten Speichels, sowie die Menge des im Augenblick der Ausheberung bereits in den Darm entleerten Mageninhaltes bekannt sein, sondern auch - worauf -meist nicht genügend hingewiesen worden ist - der Verla uf der Entleerung und der Absonderung. Denri es ist natürlich für die im Augenblick der Ausheberung erreichte Zusammensetzung und Menge des Mageninhalts keineswegs gleichgültig, ob die Entleerung des Mageninhalts in den Darm gleichmäßig erfolgt, etwa so, daß in gleichen Zeitabschnitten immer die gleiche Menge oder der gleiche Bruchteil des Vorhandenen in den Darm abgeschoben wird, ob in gleichen Zeiten immer dieselbe Menge Magensaft von konstanter Zusammensetzung abgesondert wixd, oder ob gleich nach Einführung der Probemahlzeit ein verhältnismäßig großer Teil des flüssigen Inhalts in den Darm gelangt und nachher in unregelmäßigen Zeitabschnitten wechselnde Mengen entleert werden und auch die Absonderung des Magensaftes erst allmählich qualitativ und quantitativ ihre endgültige Höhe erreicht. Es ist aber gerade sehr wahrscheinlich, daß der zuletzt genannte Modus der Entleerung und der Absonderung den tatsächlichen Verhältnissen entspricht. Zahlreiche Untersucher haben sich bemüht, eine Methode der klinischen Magenuntersuchung auszuarbeiten, die es ermöglichte, die Zusammensetzung und Menge des abgesonderten Magensaftes zu berechnen, aber ganz abgesehen davon, daß bisher wohl keine dieser Methoden allgemeiné Annahme und Verwendung gefunden hat, beruhen alle diese Methoden auf Voraussetzungen, die sicherlich nicht zutreffen. Bedenkt man endlich, daß die Entleerung des Mageninhalts in den Darm und die Absonderung des Magensaftes einer großen Zahl von wechselnden Einflüssen unterworfen sind, die unter pathologischen Verhältnissen gewiß erst recht unkontrollierbar sein werden, so sieht man ein, daß alle diese Bemühungen von vornherein zur Unfruchtbarkeit verurteilt sein müssen. Man muß sich um so mehr darüber wundern, daß es trotz dieser Schwierigkeiten den Klinikern gelungen ist, auf rein empirischem Wege aus der Untersuchung des ausgeheberten Mageninhalts ausreichende Schlüsse auf die Funktion des erkrankten Magens zu ziehen, aber man wird es verständlich finden, daß so häufig über unbegreifliche Schwankungen der Untersuchungsergebnisse geklagt wird. Durchaus unberechtigt ist es jedenfalls, aus solchen Schwankungen den Schluß zu ziehen, daß die Absonderung des Magensaftes selbst qualitativ und quantitativ einem starken Wechsel unterworfen sei; die erwähnten Verbältnisse genügen vollauf, um auch bei einer ganz gleichmäßigen Ab- 
sonderung des Magensaftes die Zusammensetzung und Menge des Mageninhaltes sehr schwankend zu gestalten.

Wir sind daher für die Kenntnis der Beschaffenheit des normalen Magensaftes zunächst ganz angewiesen auf die Versuche am Tiere, speziell am Hunde, bei dem wir nach dem von Pawlow angegebenen Verfahren (Anlegung einer Magen- und Speiseröhrenfistel, Scheinfütterung) absolut reinen Magensaft in beliebiger Menge - wie Pawlow sagt, wie Milch von der Kuh - erhalten können. Ich habe - soweit ich die Literatur übersehe, als erster und bisher einziger Untersucher - größere Mengen eines derartigen Magensaftes, der von einem im besten Emährungszustande sich befindenden Hunde bei lebhafter Sekretion der Magendrüsen gewonnen war, einer eingehenden Analyse unterworfen ${ }^{2}$ ). Danach ist die Zusammensetzung des Hundemagensaftes die folgende:

\begin{tabular}{|c|c|c|c|}
\hline Menge des untersuchter & Magensaftes. & $\begin{array}{l}\text { Versuch 1: } \\
1480 \mathrm{cem}\end{array}$ & $\begin{array}{l}\text { Versuch 2: } \\
1350 \mathrm{cem}\end{array}$ \\
\hline Trockensubstanz. . . & . . . . . . & $0,38732 \%$ & - \\
\hline Organische Substanz. & . . . . . & $0,26060 \%$ & - \\
\hline Gesamtasche . . & . . & $0,12672 \%$ & $0,13604 \%$ \\
\hline $\mathrm{HCl}$ & . . . . & $0,56570 \%$ & $0,55690 \%$ \\
\hline $\mathrm{Cl}$ in $\mathrm{HCl}$ & . . . & $0,55020 \%$ & $0,54160 \%$ \\
\hline Chloralkalien . . & ..... . & $0,12294 \%$ & $0,13265 \%$ \\
\hline Cl in Chloralkalien & . . . . & $0,06715 \%$ & $0,06958 \%$ \\
\hline$\cdot \cdot$ & . . . . . . & $0,02502 \%$ & $0,01979 \%$ \\
\hline . . & . . . . & $0,03077 \%$ & $0,04328 \%$ \\
\hline $\mathrm{SO}_{3} \ldots \ldots$ & $\cdot \cdot \cdot \cdot \cdot \cdot$ & $0,00118 \%$ & $0,00094 \%$ \\
\hline$\cdot \cdot \cdot \cdot$ & . . . . & $0,00022 \%$ & $0,00007 \%$ \\
\hline$\cdot \cdot \cdot$ & - . & $0,00049 \%$ & $0,00053 \%$ \\
\hline $\mathrm{P}_{2} \mathrm{O}_{5} \cdot \cdot$ & . & $0,00061 \%$ & $0,00068 \%$ \\
\hline
\end{tabular}

Sieht man von den nur in ganz geringfügigen Mengen vorkommenden Bestandteilen ab, so enthält also der Magensaft : $0,26 \%$ organische Substanz (vorwiegend eiweißartiger Natur), 0,56-0,57\% Salzsäure und 0,12 bis $0,13 \%$ Chloralkalien, alles andere verschwindet dagegen vollständig. Immerhin sind diese nur spurweise vorkommenden Bestandteile nicht ganz ohne Interesse. Es ist nämlich bemerkenswert, daß das Kalium das Natrium und das Magnesium das Calcium überwiegt. In den Flüssigkeiten des Körpers finden sich gerade die an zweiter Stelle genannten Metalle in größerer Menge als die an erster Stelle aufgeführten, in den festen Bestandteilen dagegen ist das Verhältnis umgekehrt, etwa so wie im Magensafte. Das scheint mir darauf hinzuweisen, daß der Magensaft nicht etwa ein einfaches Transsudat der Blut- oder Lymphflüssigkeit ist, sondern daß er aus den Zellen der Magendrüsen stammt, die ihn durch aktive Tätigkeit bereiten. Schwefelsäure und Phosphorsäure resp. die Salze dieser Säuren enthält der normale Magensaft überhaupt nicht; denn die geringen Mengen, die jch darin fand, dürften aus der Ver- 
Drennung des Eiweißes stammen. Es ist nötig, die Abwesenheit von Schwefel- und Phosphorsäure im normalen Magensafte besonders zu betonen, weil sich die entgegengesetzte Angabe mit großer Hartnäckigkeit erhält: $\left.{ }^{3}\right)$. Besonders die ,sauren Phosphate ${ }^{6}$ spielen noch immer in den Úberlegungen vieler Autoren über die Acidität des Magensaftes eine gewisse Rolle, obwohl schon mehrfach darauf hingewiesen worden ist ${ }^{4}$ ), daß ihnen sicherlich eine besondere Bedeutung dabei nicht zukommt. Sollten sich in einem normalen Mageninhalte solche Phosphate finden, so könnten sie jedenfalls nicht aus dem Magensafte, sondern nur aus der Probemahlzeit stammen, die natürlich Phosphate immer enthalten wird.

Die anorganischen Bestandteile des Mag ensaftes sind also fast ausschließlich Chlorverbindungen: Salzsäure und Chloralkalien. Die Magenschleimhaut hat ein ausgesprochenes Anziehungsvermögen für Chloride, sie speichert sie bereits vor Beginn der Verdauung in sich auf. Ich habe in besonders hierauf gerichteten Versuchen den Chlorgehalt der Magenschleimhaut von Hunden gleichen Wurfes vor und nach der Absonderung des Magensaftes untersucht und mit dem Chlorgehalt der andern Körperbestandteile verglichen ${ }^{5}$ ). Dabei hatte die Magenschleimhaut vor Beginn der Magensaftabsonderung den höchsten Chlorgehalt von allen untersuchten Körperbestandteilen, er war noch etwas höher als der des Blutes. Wäbrend der Verdauung dagegen war der Chlorgehalt der Magenschleimbaut stark herabgesetzt, und zwar war die Chlorabgabe in Prozenten der am Beginn der Verdauung vorhandenen Menge hier größer als an irgendeiner anderen Stelle des Körpers. Daraus geht deutlich hervor, daß während des Hongers eine Speicherung von Chlorverbindungen in der Magenschleimhaut stattfindet, aus denen dann während der Absonderung die Salzsäure abgespalten wird. Diese Chlorspeicherung ist ein für die Magenschleimhaut ebenso charakteristischer Vorgang wie die darauffolgende Salzsäurebildung, die Chlorspeicherung: muß geradezu als der erste Akt der Drüsentätigkeit bezeichnet werden. Dabei darf man sich aber die Dinge nicht etwa so vorstellen, als ob in der Zeit vor Beginn der Absonderung bereits die gesamte nachher erforderliche Chlormenge in der Magenschleimhaut aufgespeichert und. isozusagen bereit gehalten würde. Dafür ist das Gewicht des Organs viel zu klein und andererseits die erforderliche Chlormenge viel zu groß. Der Hauptanteil der für die Salzsäureabsonderung erforderlichen Chloride wird erst während der Absonderung selbst aus dem die Schleim"haut durchströmenden Blute resp. der Lymphe entnommen. In demselben Maße, in dem aus den aufgespeicherten Chloriden Salzsäure ąbgespalten und in den Magen ausgeschieden wird, reißen die Zellen der Magendrüsen neue Chlorverbindungen an sich. Die Gesamtmenge des: dureh den Magensaft bei einer Soheinfütterung ausgeschiedenen Chlors 
betrug in meinen Versuchen an einem $26 \mathrm{~kg}$ schweren Tiere bei lebhafter Sekretion zwischen 4,5 und $5,4 \mathrm{~g}$, während der gesamte Chlorvorrat des Körpers zwischen 27,0 und $31,0 \mathrm{~g}$ Chlor schwankte; es wurden also $15-20 \%$ des gesamten Vorrats ausgeschieden ${ }^{6}$ ). Ich habe andererseits in Versuchen, in denen der Chlorvorrat des Körpers experimentell herabgesetzt wurde ${ }^{6}$ ), nachgewiesen, daß die Magensaftsekretion bereits vollständig versiegt, wenn der Körpex noch $80 \%$ seines normalen Chlorgehalts besitzt, daß also nur etwa $20 \%$ des gesamten Chlorvorrats des Körpers für die Magensaftsekretion disponibel sind. Bei lebhaftester Sekretion wird also annähernd geradesoviel Chlor im Magensafte auisgeschieden, als überhaupt möglich ist. Wenn im Laufe der Absonderung der Körper mehr und mehr an Chlor verarmt, stellen sich der Chlorspeicherung in den Zellen der Magendrüsen offenbar immer größere Widerstände entgegen, die schließlich zum Aufhören der Sekretion führen. Dabei wird allerdings auch die Wasserverarmung des Körpers eine Rolle spielen; in meinen Versuchen betrug die Menge des abgesonderten Magensaftes bis zur Hälfte der Blutmenge 7).

Während die Chlorspeicherung in der Magenschleimhaut eine Funktion vorstellt, die offenbar dauernd während der Ruhe sich vollzieht, bis das Maximum der Chloranreicherung erzielt ist, setzt die eigentliche Absonderung, die Abgabe der Chloride im Magensafte in Form von Salzsäure und Chloralkalien erst in dem Augenblicke ein, in dem der Magensaft vom Körper gebraucht wird, und zwar auf Grund bestimmter Reize, deren Kenntnis wir vor allem Pawlow verdanken. Es sind zwei Arten solcher Reize zu unterscheiden, einmal diejenigen, die während der Aufnahme der Nahrung entstehen: psychische Vorgänge, wie das Verlangen nach Nahrung und die Befriedigung dieses Verlangens während der Nahrungszufuhr, Geschmacks- und Geruchsreize, sensible Reize von der Schleimhaut der Mundhöhle, des Rachens und der Speiseröhre, und zweitens chemische Wirkungen, welche die aufgenommenen Speisen und die im Laufe der Verdauung daraus entstehenden Produkte von der Magenschleimhaut aus auslösen. Dazu können sich gesellen hemmende Einflüsse, wie unangenehme Vorstellungen und Affekte einerseits und die eigentümliche Wirkung der Fette im Magen andererseits. Die hieraus resultierende Gesamatinnervation wird jedeñfalls einen nach den Verhältnissen schwankenden Reiz von sehr wechselnder Inteusität darstellen; ich bin davon überzeugt, worauf ich weitèr unten noch zurückkommen werde, daß von dieser Intensität des Reizes nicht nur die Menge, sondern auch die Zusammensetzung des abgesonderten Saftes sehr wesentlich abhängen wird. In der Pawlowsehen Scheinfütterung besitzen wir eine Versuchsanordnung, die es gestattet, die bei der Aufnahme der Speisen entstehenden Reizé in der größten Intensität zu erzeugen; die hierauf erfolgende Magensaftabsonderung 
dürfte daher auch das Maximum dessen darstellen, was die Magenschleimhaut unter im übrigen günstigen Verhältnissen (Wasser- und Chlorvorrat des Körpers) zu leisten imstande ist. Von den üblichen Methoden der Untersuchung der Magentätigkeit durch Probemahlzeiten beim Menschen wird man das aber leider durchaus nicht sagen können. Am ehesten dürfte es noch für die Probemittagsmahlzeit gelten, die den gewöhnlichen Verhältnissen der Nahrungsaufnahme wohl am nächsten kommt. Für das in den meisten Fällen aber angewendete Probefrühstück dürfte das keineswegs zutreffen. Eine trockne Semmel mit zwei Tassen ungesüßten Tees wird für die wenigsten Menschen eine besonders angenehme Nahrung darstellen, der bittere Geschmack des Tees wird für die, die nicht gerade an dieses Getränk gewöhnt sind, eher höchst unangenehm wirken. Das bedenklichste Moment aber, das bei allen Probemahlzeiten sich geltend machen muß, wird die Furcht des Patienten vor der ihm bevorstehenden Einführung des Magenschlauchs sein. Es ist ja bekannt, daB man sich bei häufiger Wiederholung schließlich weitgehend an diese Prozedur gewöhnen kann; in der überwiegenden Mehrzahl der Fälle wird es sich aber doch immer nur um eine einmalige oder seltener wiederholte Maßnahme handeln, bei der die Furcht vor den unangenehmen Empfindungen bei der Einführung des Magensohlauches, der Ekel vor der Entleerung des Mageninhalts, die die meisten Personen an den Brechakt erinnern wird, einen psychischen Zustand des Patienten herbeiführen muß, der für die Anregung der Magensekretion alles andere als günstig sein wird. Wie stark solche psychische Momente die Magenabsonderung beeinflussen, ist ja aus der gewöhnlichen Erfahrung bereits:bekannt und durch die Versuche Bie kels und Bönnigers $\left.{ }^{8}\right)$ noch besonders nachgewiesen worden. Man wird daher bei allen Untersuchungen der Magentätigkeit mit Hilfe einer Probemablzeit von vornherein nicht auf eine maximale Anregung der Magendrüsen rechnen dürfen, eine Tatsache, die doch wohl mehr Berücksichtigung verdient, als ihr im allgemeinen geschenkt zu werden soheint.

Wenn nun unter dem EinfluB der Sekretionsreize die eigentliche Absonderung des Magensaftes beginnt, werden die in den Magendrüsen entweder schon vorher oder im Verlaufe der Absonderung gespeicherten Chloride in den Magen abgegeben, zum Teil als Chloralkalien, zum Teil als Salzsäure. Bei meinen Versuchen ergab sich die bemerkenswerte Tatsache, daß die Gesamtmenge des im Magensafte in Form von Salzsäure + Chloralkalien abgesonderten Chlors bei günstigen. Sekretionsbedingungen nur in engen Grenzen schwankte, sie betrug 0,54-0,64\% $\mathrm{Cl}$; im Verlaufe eines einzelnen Versuchs war die Schwankung noch geringer, so daß man von einer fast völligen Konstanz des Gesamt-Cl-Gehalts sprechen kann ${ }^{9}$ ). Der Cl-Gehalt des Magensaftes ist danach doppelt so hoch wie der des Blutes und immer noch erheblich höher als der des 
Blutserums. Dies beweist wiederum, daß in den Magendrüsen ein Ansammeln, eine Aufspeicherung der Chlorverbindungen über die im Blute vorhandene Konzentration hinaus stattfindet. Die annähernde Konstanz des Gesamtchlorgehaltes des Magensaftes steht in guter Übereinstimmung mit der ebenfalls nur wenig schwankenden Gefrierpunktserniedrigung desseIben ${ }^{10}$ ); diese ist bekanntlich der beste Ausdruck für die molekulare Konzentration einer Flüssigkeit, sie kann bei der $\mathrm{Zu}$ sammensetzung des Magensaftes nur von dem Gehalt desselben an Chloriden abhängen, und es ist dafür fast ohne Bedeutung, ob die Chloride als Salzsäure oder als Chloralkalien ausgeschieden werden. Die Gefrierpunktserniedrigung des Magensaftes war in meinen Versuchen bei günstigen Sekretionsbedingungen immer etwa gleich der des Blutes, nicht selten merklich, wenn auch nur wenig größer. In der Literatur liegen allerdings auch vereinzelte Beobachtungen vor, in denen die Gefrierpunktserniedrigung des Magensaftes beträchtlich größer als die des Blutes gewesen sein soll; ich stehe aber diesen Angaben sehr skeptisch gegenüber. Ich betrachte die Tatsache, daß die Gefrierpunktserniedrigung des Magensaftẹs in meinen Versuchen auch bei lebhaftester Ábsonderung immer nur wenig über der des Blutes lag, als ein Zeichen dafür, daß der maximale Betrag der Chlorspeicherung in den Magendrüsen von den Bedingungen des osmotischen Druckes abhängt. Das Vermögen der Magendrüsen, Chlor elektiv an sich zu reißen, findet seine oberste Grenze in dem Verhältnis zwischen dem osmotischen Druck innerhalb der Zellen und im Blute; sowie der osmotische Druck in den Zellen auch nur etwas über den des Blutes hinausgeht, ist eine weitere Anreicherung an Chloriden unmöglich. Die Chlorspeicherung in den Zellen der Magendrüsen ist eine besondere Funktion dieser Zellen, die für die Zusammensetzung des weiterhin gebildeten Sekretes von maßgebender Bedeutung ist. In meinen Versuchen mit chlorarmer Ernährung war sowohl der Gesamtchlorgehalt wie die. Gefrierpunktserniedrigung des Magensaftes deutlich herabgesetzt ${ }^{11}$ ).

Bei der eigentlichen Absonderung des Magensaftes wird nun aus einem Teil der aufgespeicherten Chlorverbindungen die Salzsäure abgespalten, der andere Teil wird als Chloralkalien abgeschieden. Die Salzsäureabspaltung ist eine besondere Funktion der Magendrüsen, die von der Funktion der Chlorspeicherung scharf getrennt werden muß. Die Chlorspeicherung erfolgt ohne einen besonderen Reiz schon in der Zwischenzeit zwischen zwei Verdauungsperioden und im Verlaufe der Magensaftabsonderung, sowie eben der Chlorvorrat der Zelle sinkt. Sie ist im hohen Maße konstant und nur von dem Gesamtzustande des Körpers, vor allem seinem Wasser- und Chlorgehalt abhängig, der ja experimentell $\mathrm{zu}$ beeinflussen ist, unter den gewöhnlichen Verhältnissen des Lebens aber kaum in nennenswertem Grade schwankt. Die Salz- 
säureabspaltung dagegen kann in viel höherem Maße wechseln, sie hängt vor allen Dingen von der Größe des Sekretionsreizes ab, der, wie oben schon gesagt. wurde, sehr verschieden groß sein kann. Bei starkem Sekretionsreiz, so z. B. bei der Scheinfütterung auf der Höhe der Sekretion erscheint die Hauptmenge der Chloride des Magensaftes als Salzsäure, im Beginn und gegen Ende der Sekretion sinkt die Menge der Salzsäure unter entsprechendem Ansteigen der Chloralkalien. Ich führe für dieses sehr charakteristische Verhalten nur einen meiner Versuche (Versuch 6) an ${ }^{12}$ ):

$\begin{array}{ccccc}\text { Stunde } & \text { Menge des Magensaftes } & \begin{array}{c}\text { Gesamt-Cl } \\ \%\end{array} & \mathrm{Cl} \text { in HCl } & \mathrm{Cl} \text { in Chloralkalien } \\ 0-1 / 2 & \text { ccm } & 0 & \% \\ 1 / 2-1 & 169 & 0,56 & 0,45 & 0,11 \\ 1-1^{1} / 2 & 145 & 0,58 & 0,53 & 0,05 \\ 11 / 2-2 & 102 & 0,58 & 0,53 & 0,05 \\ 2-2^{1 / 2} & 43 & 0,56 & 0,48 & 0,08 \\ 21 / 2-3 & 21 & 0,56 & 0,32 & 0,24 \\ 3-3^{1} / 2 & 34 & 0,58 & 0,37 & 0,21 \\ 31 / 2-4 & 20 & 0,57 & 0,39 & 0,18 \\ 4-4^{1} / 2 & 21 & 0,57 & 0,34 & 0,23 \\ & 25 & 0,56 & 0,34 & 0,22\end{array}$

Für diese Schwankungen im HCl-Gehalt des Magensaftes in den verschiedenen Portionen eines Versuchs haben Heidenhain ${ }^{13}$ ) und $\mathrm{Paw}$ lo ${ }^{14}$ ) folgende Erklärung gegeben*). Sie nehmen an, daß der Magensaft stets mit einer konstanten Acidität produziert wird, daß aber nachträglich eine teilweise Neutralisation durch den alkalischen Magensehleim bedingt würde. Bei hoher Sekretionsgesehwindigkeit würde dadurch natürlich der HCl-Gehalt des Saftes viel weniger herabgesetzt als bei spärlicher Absonderung; so erkläre sich das beinahe konstante Faktum, daß die Acidität des Saftes um so höher sei, je stärker die Sekretion ist und umgekehrt. Wenn im Laufe einer lebhaften Sekretion allmählich der alkalische Magenschleim neutralisiert sei, so wäre nunmehr der Saft gleichmäßig, und zwar sehr stark sauer, einerlei, ob er schnell oder langsam abgesondert werde; beim Abklingen der Sekretion vermisse man dann die niedrige Acidität, die im Anfange des Versuchs den entsprechenden Sekretionsgeschwindigkeiten eigen war.

Diese Anschauung von der nachträglichen Neutralisation ejnes ursprünglich mit immer gleichem Säuregehalt abgesonderten Magensaftes ist von der Mehrzahl der spätexen Autoren fast wie eine bewiesene Tatsache hingenommen worden, während es sich doch nur um eine Erklärung der Beobachtungen handelt, die vielleicht auch auf andere Weise erklärt werdén köönnen und müssen. Mir erscheint die Vorstellung, daß die Acidität des abgesonderten Stoffes von vornherein verschieden groß

*) In Cer Titeratur wird diese Anschaung regelmäßig auf $P$ awlow zurückgeführt; sie ist aber bereits von Heidenhain ausgesprochen worden. 
ist, je nach der Intensität des Sekretionsreizes, zum mindesten ebenso gut denkbar und überdies mit den Tatsachen in viel besserer Übereinstimmung. Denn ich konnte bei meinen Versuchen die Angabe Pawlows, daß nur im Anfang der Sekretion die Acidität des nach arßen. entleerten Magensaftes niedriger sei als auf der Höhe der Sekretion, da $B$ dagegen beim Abklingen der Sekretion eine derartige niedrige Acidität vermißt werde, nicht bestätigen, in meinen Versuchen sank die Acidität regelmäßig gegen Ende der Sekretion, wie es das oben gegebene Beispiel deutlich zeigt. Hier hätte doch der alkalische Magenschleim durch die vorhergehende intensive Berieselung der Magenschleimhaut mit dem stark sauern Sekret der ersten $1 \frac{1}{2}$ Stunden abneutralisiert sein müssen, so daß die Acidität des nunmehr in geringerer Menge abgesonderten Saftes gleich geblieben wäre. Wenn gleichwohl in der Tat hier eine starke Abnahme der Acidität eintritt, so könnte man das, wenn man die Heidenhain-Pawlowsche Anschauung festhalten wollte, nur durch die Annahme erklären, daß gegen Ende der Sekretion die Bildung des alkalischen Magenschleims stark zugenommen babe. Für eine derartige Annahme fehlt aber doch jede tatsächliche Unterlage. Überhaupt ist die Beimischung von Magenschleim bei dem durch Scheinfütterung gewonnenen Safte nach meiner Erfahrung immer nur sehr gering und jedenfalls am Schluß des Versuchs nicht merklich größer als auf der Höhe der Sekretion. Wie groß soll man sich die "Alkalescenz" oder besser das "Säurebindungsvermögen" dieses Magenschleims vorstellen, damit sie quantitativ bei der Herabsetzung der Acidität des Magensaftes in Betracht kommen kann? Leider wissen wir ja von den chemischen Eigenschaften des Magenschleims so gut wie gar nichts: Unter pathologischen Verhältnissen kann die Schleimproduktion des Magens stark gesteigert sein, in solchen Fällen mag dann die Säurebindung durch den Schleim vielleicht mehr in Betracht kommen', ich glaube, daß man sie auch in diesen Fällen noch stark überschätzt.

Für eine Neutralisation des Magensaftes sind von anderen Autoren in Anlehnung an die Heidenhain-Pawlowsche Vorstellung noch verschiedene Flüssigkeiten herangezogen worden, so der Speichel, das alkalische Pylorussekret, der aus dem Darm in den Magen zurücktretende Pankreas-, Darm- und Gallensaft. Der Speichel kommt für die Beobachtungen bei Scheinfütterung nicht in Betracht, da ja hier der Zutritt desselben zum Magen verhindert ist; bei der Tätigkeit des Magens unter gewöhnlichen Verhältnissen könnte er aber in der Tat neutralisierend wirken. Nur würde hier die bei der Speiseaufnahme verschluckte Speichelmenge sich doch voraussichtlich ziemlich gleichmäßig dem Magensafte beimischen und so eine gleichmäBige Herabsetzung der Acidität bewirken, eine Änderung der Acidität in bestimmten Perioden der Verdauung könnte dagegen wohl kaum auf den Speichel zurück- 
geführt werden. Bedenkt man aber, wie wenig feste Bestandteile der Speichel überhaupt enthält und daß von diesen doch wieder nur der geringste Teil zur Neutralisation von Säure geeignet ist, so wird man die neutralisierende Wirkung des Speichels gewiß nicht gerade hoch veranschlagen können. Das Sekret des Pylorus ist nach Heide n hain s ${ }^{15}$ ) Angabe ,konstant alkalisch", es wird gerade gegen Ende der Verdauung in größerer Menge abgesondert, aber, wie Heidenhain ausdrücklich bemerkt, ,,die abgesonderten Mengen bleiben immerhin gering, 2-3 ccm in der Stunde". Welche Wirkung soll diese spärliche Absonderung eines Sekrets, selbst wenn es an sich stark alkalisch wäre, für die Herabsetzung der Acidität des in so reichlichen Mengen abgesonderten Magensaftes haben? Nach Pfaundler ${ }^{16}$ ) betrug der Alkalescenzgrad des. Pylorussekrets bis zu einigen Kubikzentimetern $1 / 10 \mathrm{n}$-Lauge pro $100 \mathrm{~cm}$ Substanz; das ist doch eine keineswegs hohe Alkalescenz. Was endlich den Rücktritt von Pankreas-, Darm- und Gallenflüssigkeit aus dem Darm in den Magen anlangt, so erfolgt dieser Vorgang nach Boldyreff ${ }^{17}$ ) Untersuchungen nur unter ganz besonderen Umständen: bei Einführung großer Mengen stark sauerer Flüssigkeit oder von Öl in den leeren Magen, sowie gelegentlich beim Hunger. Wollte Bold yreff während der Magenverdauung den Rücktritt der Pankreas-, Darmund Gallenflüssigkeit in den Magen herbeiführen, so war dafür nach seiner Angabe eine ,sehr viel größere Säurelösung erforderlich und es war überhaupt ungleich schwerer, diesen Übertritt hervorzurufen". Daraus ergibt sich doch wohl, daß während der normalen Verdauung ein derartiger Übertritt überhaupt nicht oder doch nur sehr ausnahmsweise erfolgen wird. Ich vermisse bei all diesen Annahmen einer Neutralisation des Magensaftes durch andere Flüssigkeiten die gebührende Berücksichtigung der quantitativen Verhältnisse. Auf der einen Seite haben wir den in reichlichen Mengen abgesonderten stark saueren Magensaft, auf der andern Seite spärliche Mengen alkalischer oder größere Mengen ganz schwach alkalischer Flüssigkeiten. Natürlich läßt sich nicht. bestreiten, daß überhaupt eine Neutralisation des Magensaftes vorkommen kann, daß sie aber quantitativ eine Rolle spielt und zur Erklärung der Schwankungen der Acidität des Magensaftes resp. Mageninhaltes ausreicht, halte ich für höchșt unwahrscheinlich.

In der Literatur findet sich sogar die Anschauung vertreten ${ }^{18}$ ), dab die Neutralisation durch ein alkalisches Sekret oder eine „Verdünnungssekretion" des Magens ein regelmäßig gegen Ende der Verdauung eintretender Regulationsvorgang sei, durch den einer zu hohen Acidität des Mageninhaltes vorgebeugt werde. Ich kann mir eine größere Verschwendung vitaler Energie nicht denken, - man entschuldige diesen unbestimmten Ausdruck bei einem Vorgang, über dessen Geschehen wir leider eben gar nichts wissen. Erst soll die Magenschleimhaut 
Salzsäure im Überschuß produzieren, eine Leistung, die doch gewiß einen erheblichen Arbeitsaufwand von den sezernierenden Zellen verlangt, dann soll sie ein alkalisches oder ein Verdünnungssekret produzieren, was natürlich nicht ohne einen entsprechenden Arbeitsaufwand geschehen kann, und das alles, damit schließlich die Wirkung des einen Sekrets durch die des anderen mehr oder weniger aufgehoben, sozusagen das Übermaß der einen Funktion durch die andere wieder korrigiert wird. Ist es da nicht viel einfacher und entspricht es nicht unseren allgemeinen physiologischen Vorstellungen viel mehr, sich vorzustellen, daß die Abspaltung der Salzsäure aus den Chloriden mit verschiedener Intensität erfolgen kann, so daß von vornherein ein Saft von derjenigen Acidität abgesondert wird, die für die Verdauungsvorgänge erforderlich und zweckmäßig ist? Der Sekretionsreiz, der bei der Aufnahme der Nahrung entsteht und sich aus einer ganzen Reihe verschiedener Faktoren zusammensetzt, wird erst in einer gewissen Zeit zu seiner maximalen Höhe ansteigen; dem entspricht die geringere Acidität des Magensaftes im Anfang der Verdauung. Ist dann die Nahrungsaufnahme beendigt, so wird der Sekretionsreiz noch eine Zeitlang unterhalten durch die chemische Wirkung der Nahrung und der aus ihr entstandenen Produkte; allmählich wird aber die Intensität des Reizes abnehmen, vielleicht auch Ermüdung der absondernden Zellen eintreten, damit wird dann die Acidität des Saftes entsprechend sinken. Ganz gut könnte man sich vorstellen, daß vielleicht auch regulatorische Einflüsse, ausgehend von der jedesmaligen Beschaffenheit des Mageninhalts, die Acidität des produzierten Saftes beeinflussen und den augenblicklichen Verhältnissen anpassen.

Auf der Höhe der Sekretionstätigkeit enthält der abgesonderte Magensaft des Hundes neben nur noch sehr geringen Mengen unzersetzter Chloralkalien $0,55-0,60 \%$ Salzsäure, fast die gesamte in der Magenschleimhaut aufgespeicherte Menge von Chlorverbindungen erscheint im Magensafte als Salzsäure. Durch eine Reihe wertvoller Beobachtungen an Menschen mit Speiseröhren- und Magenfistel ${ }^{19}$ ), an denen die Pawlowschen Scheinfütterungsversuche genau so wie am Hunde vorgenommen werden konnten, ist nachgewiesen worden, daß auch der Magensaft des Menschen einen fast gleichen Gehalt an Salzsäure haben kann. Diese U̇bereinstimmung läßt es wohl berechtigt erscheinen, wenn man auch die übrigen Ergebnisse der Hundeversuche auf die menschlichen Verhältnisse überträgt.

Danach stelle ich mir den physiologischen Vorgang der Salzsäurebildung in der Magenschleimhaut aus zwei voneinander streng zu scheidenden Prozessen zusammengesetzt vor, der Chlorspeicherung einerseits und der Abgabe der gespeicherten Chloride in das Mageninnere, verbunden mit Salzsäureabspaltung, andererseits. 
Die Chlorspeicherung erfolgt immer, solange hoch nicht der maximale Chlorgehalt der Zellen der Magendrüsen erreicht ist; also schon vor Beginn der eigentlichen Absonderung, aber auch während des Verlaufs derselben entsprechend der allmählichen Entleerung der Zellen. Die maximale Chlorspeicherung ist erreicht, wenn der osmotische Druck im Innern der Zelle den osmotischen Druck der Blutflüssigkeit eben zu übersteigen beginnt. Die Höhe der Speicherung hängt vor allen Dingen von dem Ernährungszustande ab, bei Hunger und Unterernährung, bei Wasser- und Chlorverarmung des Körpers sinkt die Menge der gespeicherten Chloride und dementsprechend nachher die Menge des im Magensafte abgeschiedenen Gesamtchlors. Setzt dann mit der Nahrungsaufnahme der Sekretionsreiz ein, so beginnt die Abgabe der aufgespeicherten Chloride in den Magen und die Abspaltung der Salzsäure daraus. Die säureabspaltende Funktion der Zellen ist in erster Linie abhängig von der Intensität des Sekretionsreizes. Bei lebhaftester Sekretion wird daher fast die gesamte aufgespeicherte Chloridmenge in Salzsäure verwandelt und in dieser Form im Magensafte ausgeschieden, ungespaltene Chloralkalien sind daneben nur in sehr geringer Menge vorhanden. Bei geringerer Sekretionstätigkeit wird ein Saft von geringerer Acidität gebildet, er enthält dann entsprechend mehr Chloralkalien. Die Menge des gesamten abgesonderten Chlors bleibt dabei unverändert, sie entspricht der Größe der Chlorspeicherung. Das Verhältnis der im Magensaft abgesonderten Salzsäure zu den daneben vorhandenen unzersetzten Chloralkalien kann als Maßstab dienen für die Größe der Sekretionsenergie der Zelle.

Unter pathologischen Verhältnissen unterscheidet man bekanntlich die folgenden Störungen der sekretorischen Funktion der Magenschleimhaut: Hyp- resp. Anacidität und Hyperacidität: Absonderung eines Magensaftes von abnorm geringer oder abnorm gesteigerter Acidität, und Hypo- und Hypersekretion: Absonderung eines qualitativ normalen Magensaftes in zu geringer oder zu großer Menge. Was nun zunächst die Hyperacidität anlangt, so haben schon $R u^{2}{ }^{20}$ ) und Bickelei) eingehend darauf hingewiesen, daß nicht eine einzige Tatsache vorliegt, die zu der Annahme nötigte, daß unter bestimmten Umständen ein Saft von abnorm hoher Acidität abgesondert werden könne. Niemals ist es bisher gelungen, weder in pathologischen Fällen beim Menschen, noch unter den Bedingungen des Experiments beim Tiere einen Saft von abnorm erhöhter Acidität, d.h. also mit einem Salzsäuregehalt von mehr als $0,6 \%$ zu erhalten. Und vom physiologischen Standpunkte aus ist es gewiß so unwahrscheinlich wie nur möglich, daß etwas Derartiges unter irgendwelchen Bedingungen vorkommen könnte. Denn auf der Höhe der normalen Sekretion ist die salzsäureabspaltende Funktion der Magendrüsen bereits, wie wir gesehen haben, so groß, 
daß so gut wie alle in der Magenschleimhaut gespeicherten Chloride zerlegt werden. Eine Steigerung des Salzsäuregehaltes des Magensaftes über die Norm wäre also nur denkbar, wenn die Chlorspeicherung einen abnorm hohen Wert annehmen könnte. Damit müßte aber natürlich auch eine entsprechende Vermehrung der osmotischen Druckdifferenz zwischen Zellinhalt und Blutflüssigkeit verbunden sein, und die Zellen müßten also die Fähigkeit gewinnen, eine derartige Druckdifferenz zu überwinden, eine Fähigkeit, die sie unter normalen Verhältnissen jedenfalls in irgendwie beträchtlichem Maße nicht besitzen. Daß gerade die geschädigte, erkrankte Zelle diese Fähigkeit gewinnen sollte, wird niemand annehmen wollen. Mit größter Bestimmtheit kann man daher behaupten, daß es eine Hyperacidität nicht gibt und nicht geben kann. Dann wäre es aber auch die höchste Zeit, endlich hieraus die Konsequenz zu ziehen und das Krankheitsbild der Hyperacidität endgültig fallen zu lassen. In der neueren klinischen Literatur findet man zwar immer häufiger die Anschauung vertreten, daß für die Annahme einer Hyperacidität keine Unterlagen vorhanden sind, daß die Ergebnisse der klinischen Magenuntersuchung, auf Grund deren man bisher die Diagnose Hyperacidität stellen zu müssen glaubte, sich ebensogut durch eine Hypersekretion mit begleitender Motilitätsstörung erklären lassen, aber zu einer radikalen Beseitigung der unter diesen Umständen geradezu irreführenden Bezeichnung Hyperacidität scheint man sich gleichwohl noch immer nicht entschließen zu können. So sagt z. B. Richartz ${ }^{22}$ ), obwohl er die Anschauung vertritt, daß es eine wahre Hyperacidität im strengen Sinne nicht gibt, gleichwohl: „Allein man wird den alteingebürgerten Ausdruck (Hyperacidität) gewissermaßen als Synonym für das korrektere ,Hypersekretion " beibehalten dürfen ". Nun braucht man sich ja gewiß nicht sonderlich darüber aufzuregen, wenn einmal für einen Begriff ein Ausdruck gebraucht wird, der nach seinem Wortsinn ihm nicht genau entspricht; das kommt ja häufig genug vor und liegt einmal im Wesen der Sprachbildung. Wenn aber durch einen unrichtigen Ausdruck immer wieder eine als falsch erkannte Vorstellung wachgerufen wird, so täte man doch wirklich im Interesse der Sache besser daran, einen derartigen Mißbrauch endgültig zu beseitigen.

Die entgegengesetzte Funktionsstörung, Hypacidität, eventuell bis. zur Anacidität gesteigert, wird bekanntlich besonders häufig bei Magenkrebs, aber auch bei einer ganzen Reihe anderer Erkrankungen des Magens oder des gesamten Organismus beobachtet. Zur Erklärung der Salzsäureverminderung ist auch hier, wie bei den Schwankungen des Salzsäuregehalts im Verlaufe der Verdauung, vielfach an eine nachträgliche Neutralisation des Magensaftes gedacht worden. So hat Reissner ${ }^{23}$ ) als ein wichtiges ursächliches Moment für den Salzsäuremangel bei Magenkrebs die Neutralisation des Magensaftes durch den aus. 
dem ulcerierten Krebs austretenden Geschwulstsaft angesehen, 'wobei er annimmt, daß auch ohne eine in die Augen fallende Erweichung und Einschmelzung des Krebses mit der Möglichkeit des Austritts von Gewebssaft zu rechnen sei. Die Möglichkeit eines derartigen Vorgangs ist natürlich zuzugeben; daß er aber für eine ausreichende ErkJärung des Salzsäuremangels. bei Magenkrebs kaum in Betracht kommen kann, ergibt sich sofort, sowie man wieder die quantitativen Verhältnisse ins Auge faßt. Welche Mengen von „Geschwulstsaft" würden erforderlich sein und wie hoch müßte man die Alkalescenz desselben annehmen, damit bei einer auch nur annähernd normalen Magensaftabsonderung der Zustand der Anacidität, wie er so häufig bei Magenkrebs gef̦unden wird, durch Neutralisation herbeigeführt werden könnte?

Als ein weiteres ursächliches Moment für die Abnahme der Salzsäure im Magensafte wird die Verschlechterung des allgemeinen Ernährungszustands des Körpers, im besondern die Chlorverarmung aufgeführt. Die Abhängigkeit der Magensaftsekretion vom Ernährungszustand des Tieres trat in meinen Versuchen sehr deutlich hervor. In denjenigen Versuchen, in denen das Körpergewicht des Tieres infolge reichlicher Ernährung hoch war (über $24 \mathrm{~kg}$ ), betrug die stündliche Saftproduktion $229-270 \mathrm{ccm}$, bei einem Körpergewicht von $21-23 \mathrm{~kg}$ dagegen nur $92-138 \mathrm{ccm}$. Und häufig war bei einer quantitativ reichlichen Saftproduktion auch der Salzsäuregehalt des Saftes sowie die Gefrierpunktserniedrigung etwas größer als bei spärlicher Absonderung. Als ich meinen Hund 10 Tage lang hungern ließ24) - nur Wasser wurde dem Tiere durch die Magenfistel zugeführt —, sank die beim Scheinfütterungsversuch abgesonderte Magensaftmenge von $782 \mathrm{ccm}$ auf $417 \mathrm{ccm}$, der Salzsäuregehalt von 0,57 auf $0,52 \%$, der Gehalt an Gesamtchlor blieb unverändert $=0,63 \%$. Ganz entsprechend fand Rütime yer ${ }^{25}$ ) bei einer Hungerkünstlerin nach einem 24tägigen Hungern die Magensaftabsonderung auf ein Probefrühstück hin normal, nur bestand eine leichte Subacidität. Eine sehr viel intensivere Einwirkung auf die Magensaftabsonderung läßt sich durch die Chlorverarmung des Körpers erzielen; nur ist eine solche Chlorverarmung sehr viel schwerer tatsächlich zu erreichen, als man sich wohl meist vorstellt. Durch eine sog. chlorarme Ernährung gelangt man kaum zum Ziel. Denn selbst eine ausgesprochen chlorarme Nahrung enthält noch immer nicht unerhebliche Mengen Chlor; andererseits stellt der Körper unter diesen. Umständen sehr bald die Chlorabgabe im Harn so gut wie ganz ein und hält so das Chlor, das offenbar einen äußerst lebenswichtigen Bestandteil für ihn darstellt, mit großer Energie zurück. Die chlorärmste Ernährung ist ja offenbar der. Hungerzustand, bei dem wirklich gar kein Chlor in den Körper eingeführt wird. In einer 10 tägigen Hungerperiode gab mein Hund nur $0,53 \mathrm{~g}$ Chlor im ganzen im Harn ab, der prozentische Chlor- 
gehalt des Körpers war danach sogar erhöht, da das Körpergewicht in viel stärkerem Maße abgenommen hatte. Im Hunger werden eben gerade die festen Bestandteile des Körpers eingeschmolzen, die wenig oder gar kein Chlor enthalten, während der Flüssigkeitsbestand und mit diesem der Chlorgehalt relativ zunimmt. Einen merklichen Chlorverlust kann man nur dadurch herbeiführen, daß man bei geringer oder fehlender Chlorzufuhr zugleich eine Sekretion lebhaft anregt, durch die Chlor aus dem Körper entfernt wird. Das kann z. B. geschehen durch die Schweißabsonderung; Simon ${ }^{26}$ ) hat gezeigt, daß man durch intensives Schwitzen sowohl die Menge als auch die Acidität des Magensaftes herabsetzen kann. Noch wirksamer dürfte in dieser Hinsicht die Anregung der Magentätigkeit sein, die wir ja beim Tier durch die Scheinfütterung herbeifübren können. Leitet man bei einem hungernden Tier den bei einer Scheinfütterung produzierten Magensaft nach außen ab, so ergiebt sich ein starker Chlorverlust für den Körper*), der schließlich zu sehr schweren Störungen der Magentätigkeit führt. Bei meinem Versuchstiere ${ }^{24}$ ) wurden am 10. Hungertage durch eine Scheinfütterung dem Körper 2,63 $\mathrm{g}$ Cl entzogen und dadurch der Gesamtchlorvorrat auf $88,9 \%$ des Anfangswertes herabgesetzt. Bei einem nochmaligen Scheinfütterungsversuch am folgenden Tage war der Hund trotz des vorhergegangenen 10 tägigen Hungers nicht dazu zu bringen, feste Nahrung aufzunehmen, rohes, gekochtes Fleisch, Brot, auch warme Bouillon wurden verweigert. Aus der Magenfistel flossen $87 \mathrm{ccm}$ einer rein blutigroten Flüssigkeit, die sich bei spektroskopischer Untersuchung als $\mathrm{O}$-Hb-haltig erwies. In einem andern Versuch ${ }^{27}$ ), in dem nach längerer chlorarmer Ernährung durch zwei Scheinfütterungen der Chlorgehalt des Tieres auf $79 \%$ des Anfangswertes herabgedrückt worden war, kam ebenfalls die Magensekretion völlig zum Stillstand, auch dadurch, daß direkt, $500 \mathrm{~g}$ gehacktes Fleisch in den Magen eingeführt wurde, konnte keine Sekretion ausgelöst werden, nach $3 / 4$ Stunden lag das Fleisch noch unverändert im Magen. Diese Versuche zeigen, in wie hohem Grade die sekretorische Tätigkeit des Magens von dem Chlorvorrat des Körpers abhängig ist ${ }^{28}$ ). Aber man wird darüber nicht im Zweifel sein können, daß eine Chlorverarmung, die der hier experimentell herbeigeführten auch nur annähernd an die Seite gestellt werden könnte, beim Menschen weder unter normalen noch pathologischen Verhältnissen jemals eintreten wird. Selbst bei der kümmerlichsten Ernährung wird immer ein gewisses $\mathrm{Maß}$ von Chlorverbindungen in den Körper eingeführt werden und zu stärkeren Chlorverlusten fehlt jede Veranlassung. Ich komme daher zu dem Schlusse, daß bei Hyp- und Anacidität Herabsetzung deș allgemeinen Ernährungszustandes und Chlorver-

*) Richartz ${ }^{22}$ ) hat dieses Verfahren auch beim Menschen zur Bekämpfung der Hypersekretion mit gutem Erfolge angewandt.

Virchows Arehiv. Bd. 229. 
armung kaum je eine irgendwie wesentliche Rolle bei der Entstehung des Salzsäuremangels spielen werden.

So bleibt nur die eine Annahme übrig - die eigentlich von vornherein als die nächstliegende erscheinen sollte ${ }^{29}$ ) - daß die wesentliche Ursache für den Salzsäuremangel die Erkrankung der Magendrüsen darstellt, daß die durch Krankheit geschädigte Drüse eben einen Saft von geringerem Salzsäuregehalt ausscheidet. Dieser Auffassung scheint die Heidenhain-Pawlowsche Annahme, die Drüsen sonderten immer einen Saft von konstanter Acidität ab, im Wege gestanden zu haben; es ist mir aber unzweifelhaft, daß diese Annahme nicht einmal für die gesunde Drüse gilt, wie ich oben ausgeführt habe, daher erst recht nicht unter pathologischen Verhältnissen. Von mehreren Untersuchern ${ }^{30}$ ) sind degenerative Veränderungen in den Magendrüsen bei Hyp- und Anacidität mikroskopisch nachgewiesen worden, Hammerschlag and Matti behaupten sogar, daß eine vollständige Übereinstimmung bestehe zwischen dem chemischen und histologischen Befunde; andere Untersucher vermißten allerdings eine so weitgehende Übereinstimmung. Es wäre ja auch nichts Außergewöhnliches, wenn die Erkrankung der Drüsen sich bereits zu einer Zeit in der Zusammensetzung des abgesonderten Saftes zu erkennen, geben würde, zu der eine sichtbare Veränderung der Drüse noch nicht nachweisbar wäre. Die krankhafte Veränderung der Drüsenzellen könnte nun sehr wohl die beiden Funktionen, die bei der Magensaftabsonderung, wie ich oben gezeigt habe, in Frage kommen, getrennt betreffen; es könnte entweder die Chlorspeicherung oder die Salzsäureabspaltung geschädigt sein, in andern Fällen vielleicht auch beides zu gleicher Zeit. Wäre nur die chlorspeichernde Eigenschaft der Drüse beeinträchtigt, so würde der Gesamtchlorgehalt des abgesonderten Sekretes erniedrigt sein müssen, also sowohl die Salzsäure wie die ungespaltenen Chloralkalien. Bei einer Herabsetzung der salzsäureabspaltenden Funktion wïurde der Gesamtchlorgehalt unverändert bleiben, nur der Gehalt des Saftes an Salzsäure würde erniedrigt sein, der an Chloralkalien aber entsprechend gesteigert. Daß derartige Unterschiede in der Tat überhaupt vorkommen, zeigen meine Scheinfütterungsversuche bei Hunger und bei Verminderung des Chlorvorrates des Körpers sehr deutlich. Setzt man experimentell den Chlorvorrat des Körpers herab, so muß natürlich darunter die Chlorspeicherung in den Zellen der Magendrüsen leiden, der unter diesen Umständen abgesonderte Magensaft zeigte in meinen Versuchen nicht nur eine Herabsetzung des Salzsäuregehalts, sondern auch der Menge der ungespaltenen Chloralkalien. Es war also der Gehalt an Gesamtchlor. verringert und dementsprechend auch die Gefrierpunktserniedrigung kleiner als die des Blutes. Durch den Hunger allein wurde dagegen, wie es die oben mitgeteilten Ergebnisse des Scheinfütterungsversuches am 
10. Hungertage zeigen, nur der Salzsäuregehalt des Saftes herabgesetzt, der Gehalt an Gesamtchlor blieb unverändert, so daß die Menge der ungespaltenen Chloralkalien gegen die Norm vermehrt war. Hier hatte also bei unveränderter Chlorspeicherung nur die salzsäureabspaltende Funktion der Zellen gelitten. Ich habe den Eindruck, daß von den beiden hier in Frage kommenden Funktionen der Zellen die Chlorspeicherung einer Veränderung den größeren Widerstand entgegensetzt, während die Salzsäureabspaltung leichter zu beeinträchtigen ist. Bei den irn Verlaufe der normalen Sekretion zu beobachtenden Schwankungen in der Zusammensetzung des Saftes blieb der Gesamtchlorgehalt annähernd konstant, während die Menge der Salzsäure am Beginn und am Ende der Sekretion sank. Reissner ${ }^{23}$ ) hat darauf aufmerksam gemacht, daß der bei Magenkrebs abgesonderte Saft häufig bei vermindertem Salzsäuregehalt eine Erhöhung der Menge der Chloralkalien aufweist. Er faßt diese Tatsache allerdings als ein Zeichen nachträglich stattgefundener Neutralisation des Saftes auf, mir ist es viel wahrscheinlicher, daß es sich dabei um eine getrennte Schädigung gerade der salzsäureabspaltenden Funktion der Zellen handelt. Bickel ${ }^{29}$ ) beobachtete bei einem subchronischen Magenkatarrh, der bei einem Hunde mit „,kleinem Magen“ durch Verätzung der Schleimhaut mittelst Alkohols erzeugt war, eine Herabsetzung des HCl- und Cl-Gehalts des Magensaftes; hier würde es sich also bei einer direkten schweren Schädigung der Drüsenzellen um eine Beeinträchtigung der Chlorspeicherung gehandelt haben. Es würde sicherlich eine Vertiefung unserer Kenntnisse bedeuten, wenn in den Fällen mit verringerter Salzsäureabsonderung dem Verhalten der ungespaltenen Chloralkalien mehr Aufmerksamkeit als bisher geschenkt werden würde.

Die Menge des abgesonderten Magensaftes schwankt schon bei den Versuchen am normalen Tiere in ziemlich weiten Grenzen, hier dürfte der Ernährungszustand und der Wassergehalt des Körpers vor allem eine Rolle spielen. Daß es unter pathologischen Bedingungen je nachdem zu Hypo-oder Hypersekretion kommen kann, ist danach leicht verständlich. Ich glaube aber, daß der in abnorm geringer oder abnorm großer Menge abgesonderte Saft meist auch in seiner Zusammensetzung $A b$ weichungen zeigen wird. In meinen Tierversuchen war bei quantitativ geringer Saftproduktion häufig auch der Gehalt an Salzsäure und auch an Gesamtchlor herabgesetzt. In den Fällen von pathologisch gesteigerter Saftabscheidung wird man aber keineswegs auch einen erhöhten Salzsäuregehalt des Saftes annehmen dürfen, wie ich oben schon auseinandergesetzt habe, der Saft bei Hypersekretion dürfte höchstens den normalen, vielleicht sogar einen etwas herabgesetzten Salzsäuregehalt aufweisen. Es ist von Illow a $\mathrm{y}^{\mathbf{3 1}}$ ) darauf hingewiesen worden, daß bei der sog. ,Hyperacidität“, die eben nur eine durch Störungen der 
Magenmotilität komplizierte Hypersekretion darstellt, das spezifische Gewicht des ausgeheberten Mageninhalts auffallend niedrig ist. Der normale Magensaft hat schon ein sehr niedriges spezifisches Gewicht: 1002-1004 ${ }^{32}$ ), vielleicht handelt es sich in diesen Fällen um eine wässerige Absonderung von besonders geringer Konzentration.

Außer der Salzsäure werden im pathologischen Mageninhalte häufig auch organische Säuren, vor allem Milchsäure angetroffen. Während man früher die Milchsäure als ein Produkt der Tätigkeit der Magendrüsen auffaßte und ihr auch bei der Entstehung der Salzsäure eine wichtige Rolle zuzuschreiben geneigt war, gilt sie heute allgemein als ein zufälliger Bestandteil des Mageninhalts, der mit der Drüsentätigkeit in gar keinem Zusammenhang steht. Soweit sie nicht aus den mit der Probemahlzeit aufgenommenen Speisen stammt - das bei dem Probemittagessen aufgenommene Fleisch $m u ß$ ja jedesmal Fleischmilchsäure enthalten —, wird sie als ein Produkt der Gärungserreger angesehen, die besonders bei Salzsäuremangel im Magen die günstigsten Bedingungen für ihre Entwicklung vorfinden. Mehrfach ist auch die im Filtrat des ausgeheberten Mageninhalts enthaltene Milchsäure durch Darstellung des Zinksalzes und Bestimmung des Wassergehaltes der Krystalle als Gärungsmilchsäure identifiziert worden ${ }^{33}$ ). Im normalen Hundemagensafte meiner Versuche konnte ich Milchsäure niemals nachweisen ${ }^{34}$ ). Danach könnte die Herkunft der Milchsäure als endgültig entschieden erscheinen. Ich bin mir wohl bewaßt, wie mißlich es ist, eine Frage, die nach allgemeiner Utbereinstimmung als erledigt gilt, wieder anzuschneiden; ich möchte aber gleichwohl, wenn auch mit allem Vorbehalt, die Vermutung äußern, daß unter gewissen Umständen vielleicht doch ein Teil der im Magen gefundenen Milchsäure aus den Magendrüsen stammen könnte. Ich werde zu dieser Vermutung veranlaßt durch die Ergebnisse meiner Untersuchungen über die Wasserstoffionenkonzentration des Magensaftes ${ }^{35}$ ). Ich habe bei meinen durch Scheinfütterung gewonnenen Magensäften, bei denen also jede Verunreinigung durch Speisebestandteile ausgeschlossen war, die Wasserstoffionenkonzentration elektrometrisch bestimmt, daraus kann man unter Berücksichtigung des Dissoziationsgrades der Salzsäure die zugehörigen Salzsäurewerte berechnen. Vergleicht man diese mit den Werten, die durch Titration mit 0,1 norm. Natronlauge und Phenolphthalein gewonnen worden sind, so erweisen sich die letzteren regelmäßig als höher; dasselbe Verhalten haben auch schon Fraenckel ${ }^{36}$ ) und Tangl ${ }^{37}$ ) beobachtet. Bei den Magensäften mit hoher Wasserstoffionenkonzentration und hohem Salzsäuregehalt ist die Differenz fast verschwindend, sie wächst aber bei den Magensäften mit niedrigeren Werten der Wasserstoffionenkonzentration und des Salzsäuregehalts und erreicht bei den unter abnormen Verhältnissen abgesonderten Magensäften sehr erheb- 
liche Beträge. Wenn man annehmen könnte, daß die durch Titration bestimmte Acidität nicht einzig und allein durch Salzsäure gebildet würde, sondern daß neben der Salzsäure noch eine andere schwache, d. h. wenig dissoziierte Säure, z. B. Milchsäure im Magensafte vorhanden sei, und zwar um so mehr, je weniger Salzsäure vorhanden ist, so wäre alles erklärt. Man kann gegen eine solche Vermutung nicht einwenden, daß ja die im Mageninhalte aufgefundene Milchsäure als Gärungsmilchsäure erkannt worden ist, während eine in den Magendrüsen entstehende Milchsäure doch natürlich höchstwahrscheinlich Fleischmilchsäure sein müßte. Denn es ist selbstverständlich zuzugeben, daß die Milchsäure im pathologischen Mageninhalte in der Mehrzahl der Fälle aus Gärungsvorgängen stammt; das schließt aber nicht aus, daß vielleicht in gewissen Fällen daneben auch eine geringe Menge Fleischmilchsäure vorhanden sein könnte, deren Nachweis bisher nicht gelungen ist. Nimmt man einmal als möglich an, daß auch die Magendrüsen unter bestimmten Bedingungen, so vor allem bei herabgesetzter Sekretionsenergie, Milchsäure bilden können, so würde man doch wohl zunächst ihre Entstehung mit Kohlehydraten in Zusammenhang bringen. Nun besteht in der Tat ein allerdings noch recht unklarer Zusammenhang zwischen Kohlehydraten und Magensaftabsonderung. Hasselbalch ${ }^{38}$ ) hat gezeigt, da $\beta$ die regelmäßigen Schwankungen in dem Wasserstoffionengehalt des Harns während der Verdauung vollständig verschwinden, wenn man eine kohlenhydratfreie Diät einhält. Ich habe die Wasserstoffionenkonzentration des Harns in ihrem Verlaufe während des Tages in meinem Institut durch Frl. Rannenberg*) eingehend untersuchen lassen; es zeigte sich dabei, daß die Schwankungen der Harnreaktion keineswegs in einem so einfachen Verhältnis zur Verdauung stehen als man gewöhnlich wohl annimmt, aber auch sie konnte zeigen, daß bei Hunger, ebenso aber auch bei kohlenhydratfreier Diät diese Schwankungen vollständig in Wegfall kommen. Was das zu bedeuten hat, läßt sich allerdings zur Zeit noch nicht sagen; jedenfalls weisen diese Befunde auf eine Beziehung zwischen Magensaftabsonderung und Kohlenhydraten hin, die vielleicht mit der Bildung von Milchsäure aus Kohlenhydraten zusammenhängen könnte. Ich habe die Schleimhaut des Schweinemagens vergebens auf Glykogen untersucht; dies negative Ergebnis könnte ja aber sehr wohl auf einer postmortalen Zersetzung etwa vorhandenen Glykogens beruhen. Mein Assistent, Herr Dr. Seidel hat allerdings in der Schleimhaut des Schweinemagens regelmäßig Milchsäure nachweisen können **); es ist aber natüxlich zunächst auch nicht zu sagen, ob es sich dabei um eine Milchsäurebildung während des Lebens handelt. Schließlich müßte man auch daran denken, daß die neben Salzsäure ver-

*) Die Versuche werden in nächster Zeit veröffentlicht werden.

**) Noch nicht veröffentlichte Untersuchungen. 
mutete schwache Säure eine andere Säure als gerade Milchsäure sein könnte. In dieser Hinsicht ist es bemerkenswert, daß v. Tabora ${ }^{39}$ ) im säurefreien Magensaft größere Mengen von Phosphaten als in der Norm vorgefunden hat. Er berichtet von einem Fall, in dem die HCl-Produktion anfangs noch gut war, die Phosphatmenge gering; allmählich sanken die Werte für freie HCl, und als diese vollständig fehlte, betrug der Phosphatwert mehr als das Doppelte des Anfangs. Ich meine, daß man eine derartige Beobachtung doch überhaupt nicht anders auffassen kann, als durch die Annahme einer Absonderung von Phosphaten durch die erkrankte Magenschleimhaut. Wenn auch im normalen Magensafte sicherlich Phosphorsäure ebensowenig wie Milchsäure vorkommt, so könnte das unter pathologischen Verhältnissen ja sehr wohl anders sein. Alle diese Fragen bedürfen und verdienen noch eingehende Untersuchung. Leider machen es die heutigen Verhältnisse fast unmöglich, Versuche an Magenfistelhunden anzustellen, wegen der schwierigen Ernährung dieser Tiere. Sonst würden Scheinfütterungsversuche bei experimentell herabgesetzter Magensaftsekretion hierfür die reinsten Verhältnisse darbieten. Inzwischen könnte doch auch die Untersuchung des menschlichen Mageninhalts in Fällen von herabgesetzter Magensaftsekretion verschiedenen Ursprungs vielleicht manchen Aufschluß bringen, wenn man der Natur der vorhandenen Milchsäure mehr Beachtung schenkte und eventuell auch auf Phosphorsäure untersuchte. Wenn nach einem Probefrühstück bei vorher sicher leerem oder rein gespültem Magen schon nach einer Stunde im Mageninhalte erhebliche Mengen von Milchsäure vorhanden sind, so erscheint es mir doch recht fraglich, ob diese Zeit ausreicht, um soviel Milchsäure allein durch Gärung zu bilden, wenn auch die Bedingungen für die Lebenstätigkeit der Milchsäurebakterien im kranken Magen offenbar besonders günstig sind. Zunächst müssen doch die Kohlenhydrate der Semmel saccharifiziert werden, ehe'die Tätigkeit der Milchsäurebildner beginnen kann. Solche Fälle verdienten meiner Meinung nach ganz besonders eine sorgfältige Untersuchung der Art der vorgefundenen Milchsäure. Sollte es etwa gelingen, in der Tat nachzuweisen, daß unter bestimmten Bedingungen dennoch entgegen der allgemeinen heutigen Vorstellung in den Magendrüsen noch andere Säuren wie nur Salzsäure gebildet werden, so würde das sicherlich für unsere jetzt noch ganz unklaren Vorstellungen von dem Vorgang der Salzsäurebildung in den Zellen der Magendrüsen von größter Bedeutung sein.

\section{Zusammenfassung.}

1. Bei der Säureabsonderung der Magensehleimhaut sind zwei Vorgänge zu unterscheiden: die Chlorspeicherung und die Säureabspaltung. Die Chlorspeicherung erfolgt sowohl in der Ruhe wie während der Magen- 
saftabsonderung, so lange noch nicht der maximale Chlorvorrat der Drüsenzelle erreicht ist, das Maximum der Chlorspeicherung hängt von dem osmotischen Druck des Blutes ab. Die Säureabspaltung schwankt nach der Größe des Sekretionsreizes, bei intensivster Sekretion wird fast die gesamte Menge des aufgespeicherten Chlors in Form von Salzsäure in den Magen abgeschieden, bei geringerem Sekretionsreiz sondern die Drüsen einen Saft von geringerem Gehalt an Säure und entsprechend vermehrtem Gehalt an unzerlegten Chloriden ab.

2. Eine Hyperacidität, d. h. Absonderung eines Saftes von höherem Salzsäuregehalt als normal $(0,55-0,60 \%)$ gibt es nicht.

3. Bei Hyp- und Anacidität sondern die erkrankten Drüsenzellen von vornherein einen Saft von geringerem. Säuregehalt als normal ab; eine nachträgliche Neutralisation des Saftes durch alkalische Flüssigkeiten spielt bei dem Zustandekommen des Salzsäuremangels keine wesentliche Rolle. Es kann entweder die Chlorspeicherung herabgesetzt sein: dabei ist sowohl der Salzsäure- wie der Gesamt-Cl-Gehalt des Saftes verringert, dies scheint der seltnere Fall zu sein — oder die Säureabspaltung ist geschädigt, was leichter einzutreten scheint: dann ist der Salzsäuregehalt des Saftes verringert, aber der Gesamt-Cl-Gehalt unverändert, die Menge der unzerlegten Chloralkalien daher entsprechend erhöht.

4. Es wird die Vermutung ausgesprochen, daß bei herabgesetzter Magensaftsekretion vielleicht doch, entgegen der heutigen Anschauung, von den Magendrüsen noch andere Säuren als Salzsäure (Milchsäure, Phosphorsäure) in dem Sekrete abgeschieden werden könnten.

\section{Literaturverzeichnis.}

1) Pawlow, I. F., Die Arkieit der Verdauungsdrüsen. Deutsch von A. Walther. Wiesbaden 1898. - ${ }^{2}$ ) Rose mann, R., Beiträge zur Physiologie der Verdauung. 1. Mitteilung. Die Eigenschaften und die Zusammensetzung des durch Scheinfütterung gewonnenen Hundemagensaftes. Arch. f. d. ges. Physiol. 118, 467. 1907. - 3) Modrakowski, G., Physiologie der Magenverdauung in L. Mohr und R. Staehelin, Handbuch der inneren Medizin. 3, 1. Teil, 340. 1918. 4) Moritz, F., Die Verdeckung der Salzsäure des Magensaftes durch Eiweißkörper. Dtsch. Arch. f. klin. Med. 44, 277. 1889; Volhard, F., Über das Alkalibindungsvermögen und die Titration der Magensäfte. Münch. med. Wochenschr. 50, 2185. 1903; v. Tabora, Über die Phosphate des Mageninhaltes. Zeitschr. f. klin. Med. 56, 369. 1905. - ${ }^{5}$ ) Rose mann, R., Beiträge zur Physiologie der Verdauung. 6. Mitteilung. Über die Chlorspeicherung in der Magenschleimhaut und die Quelle des im Magensafte abgesonderten Chlors. Areh. f. d. ges. Physiol. 168, 609. 1917; vgl. Ló pez-Suárez, J., Zur Kenntnis der Salzsäurebildung im Magen. Biochem. Zeitschr. 46, 490. 1912. - ${ }^{6}$ ) Rosemann, R., Beitr. z. Physiol. der Verdauung. 3. Mitteilung. Die Magensaftsekretion bei Verminderung des Chlorvorrates des Körpers. Arch. f. d. ges. Physiol. 142, 208 (233). 1911. 7) Nr. 2, S. 475. - $\left.{ }^{8}\right)$ Bickel, A., Experiment. Untersuch. über den Einfluß von Affekten auf die Magensaftsekretion. Dtsch. med. Wochenschr. 31, 1829. 1905; 
Bönniger, M., Magenfunktion und Psyche. Dtsch. med. Wochenschr. 39, 890. 1913. - 9) Nr. 2, S. 509 und 520. - 10) Nr. 2, S. 480. - ${ }^{11}$ ) Nr. 6, S. 216. 12) Nr. 2, S. 517. - 13) Heidenhain, R., Über die Absonderung der Fundusdrüsen des Magens. Arch. f. d. ges. Physiol. 19, 148 (165). 1879; Hermann, L., Handbuch der Physiologie, 5, 1. Teil, 158. 1883. - ${ }^{14}$ ) Nr. 1, S. 38. - ${ }^{15}$ ) Heidenhain, R., Über die Pepsinbildung in den Pylorusdrüsen. Arch. f. d. ges. Physiol. 18, 169 (171). 1878. - 16) Pf a undler, M., Über eine neue Methode zur klin. Funktionsprüfung des Magens unri deren physiolog. Ergebnisse. Dtseh. Arch. f. klin. Med. 65, 255 (281). 1900. - 17) Bold yreff, W., Der Übertritt des natürlichen Gemisches aus Pankreassaft, Darmsaft und Galle in den Magen. Arch. f. d. ges. Physiol. 121, 13 (36). 1908. - ${ }^{18}$ ) Mintz, S., Zur Frage des Chemismus des Magens. Dtsch. Arch. f. klin. Med. 104, 481. 1911; vgl. Ortner, A., Ein Beitrag zur Kenntnis der Magenentleerung und ihrer Beziehung zur Verdünnungssekretion des Magens. Arch. f. d. ges. Physiol. 168, 124 (134). 1917. 19) Sommerfeld, Zur Kenntnis der Sekretion des Magens beim Menschen. Arch. f. Physiol. 1905, Suppl. 455; Bickel, A., Experim. Untersuchungen über den Magensaft. Berl. klin. Wochenschr. 42, 60. 1905; Experiment. Untersuchungen über die Magensaftsekretion beim Menschen. Dtsch. med. Wochenschr. 32, 1323. 1906; Verhandl. d. 23. Kongr. f. innere Med. 1906, S. 481; U m ber, Die Magensaftsekretion d. (gastrostomierten) Menschen bei „Scheinfütterung“" $\mathbf{u}$ Rectalernährung. Berl. klin. Wochenschr. 42, 56. 1905; Kaznels on, H., Scheinfütterungsversuche am erwachsenen Menschen. Arch. f. d. ges. Physiol. 118, 327. 1907; Bogen, H., Experim. Untersuch. über psych. u. assoziative Magensaftsekretion b. Menschen. Arch. f. d. ges. Physiol. 11\%, 150. 1907. - $\left.{ }^{20}\right)$ Rubow, V., Die Hyperacidität des Magensaftes und ihre Bestimmung mittels der Sallischen Probemahlzeit. Arch. f. Verdauungskrankh. 12, 1, 1906, - ${ }^{21}$ ) Bickel, A., Experim. Untersuch. über die Magensaftsekretion beim Menschen. Dtsch. med. Wochenschr. 32, 1323. 1906; Verhandl. d. 23. Kongr. f. innere Med. 1906, S. 481. - ${ }^{22}$ ) Richartz, H. L., Zur Frage der Chlorentziehung bei Hypersekretion des Magens. Dtsch. med. Wochenschr. 38, 697. 1912. - ${ }^{23}$ ) Reiss ner, O., Über das Verhalten des Chlors im Magen und die Ursache des HCl-Mangels bei Magenkrebs. Zeitschr. f. klin. Med. 44, 71. 1902. - ${ }^{24}$ ) Nr. 6, S. 225. - ${ }^{25}$ ) Rütime yer, L., Über den Einfluß einer 24 tägigen Hungerperiode auf die Magensaftsekretion beim Menschen. Zentralbl. f. inn. Med. 30, 233. 1909. - ${ }^{26}$ ) Simon, A., Über den Einfluß des künstl. Schwitzens auf die Magensaftsekretion. Zeitschr. f. klin. Med. 38, 140. 1899. - ${ }^{27}$ ) Nr. 6, S. 217. - 28) Vgl. Wohlgemuth, Arbeiten aus dem patholog. Institut in Berlin. 1906. Batke, V., Über den Einfluß des Kochsalzhungers auf die Magenverdauung und über die Möglichkeit des Ersatzes der $\mathrm{HCl}$ durch $\mathrm{HBr}$ im Magen. Arch. f. d. ges. Physiol. 168, 89. 1917. - ${ }^{29}$ ) Vgl. Umber, Bogen, Nr. 19. Bickel, A., Zur patholog. Physiologie des Magenkatarrhs. Dtsch. Arch. f. klin. Med. 89, 34. 1906; Kast, L., Experiment. Beitr. zur Wirkung des Alkohols auf den Magen. Arch. f. Verdauungskrankh. 12, 487 (498). 1906; Mintz, S., Zur Frage des Chemismus des Magens. Dtsch. Arch. f. klin. Med. 104, 481. 1911. - ${ }^{30}$ ) Ham merschlag, A., Untersuchungen über das Magencarcinom. Arch. f. Verdauungskrankh. 2, 1. und 198. 1896; Matti, H., Beitr. z. Kenntnis des Magencarcinoms. Untersuchung. über die Ursachen d. veränderten Chemismus bei Fällen von Magenkrebs. Dtsch. Zeitschr. f. Chir. 104, 425. 1910; Crä mer, Über die Veränder. der Magenschleimh. bei Ulcus ventric. rotund. Arch. f. Verdauungskrankh. 17, 1. 1911; Chessin, B., Zur Frage der Gastritis bei Ulcus ventriculi. Arch. f. Verdaungskrankh. 18, 523. 1912; Heyrovsky, H., Histol. Untersuch. der Magenschleimhaut bei Ulcus ventriouli und Carcinom. Dtsch. Zeitschr. f. Chir. 122, 359. 1913. - ${ }^{31}$ ) Illowa y, H., Hyperacidität. Arch. f. Verdauungskrankh. 8, 103. 1902; vgl. Stra uss, 
H., Über das spezif. Gewicht $u$. den Gehalt des Mageninhaltes an rechtsdrehender Substanz sowie über das Verhalten der HCl-Sekretion bei Darreichung von Zuckerlösungen. Zeitschr. f. klin. Med. 29, 221. 1896. - ${ }^{32}$ ) Nr. 2, S. 479. - $\left.{ }^{33}\right)$ Cahn, A., und v. Mering, I., Die Säuren des gesunden und kranken Magens. Dtsch. Arch. f. klin. Med. 39, 233. 1886; Pfa undler, M., Nr. 16, S. 269. - ${ }^{34}$ ) Nr. 2, S. 502. - 35) Rosemann, R., Beitr. z. Physiol. der Verdauung. 7. Mitteilung. Die Wasserstoffionen-Konzentration des Magensaftes. Arch. f. d. ges. Physiol. 169, 188. 1917. - 36) Fraenckel, P., Die H-Konzentration des reinen Magensaftes und ihre Beziehungen zur elektr. Leitfähigkeit u. zur titrimetrischen Acidität. Zeitschr. f. experim. Pathol. u. Ther. 1, 431. 1905. - ${ }^{37}$ ) Tang1, F., Untersuchungen über d. Hydrogenionen-Konzentration im Inhalt des nüchternen menschlichen Magens. Arch. f. d. ges. Physiol. 115, 64. 1906. - 38) Hasselbalch, K. A., Ammoniak als physiologischer Neutralitätsregulator. Biochem. Zeitschx. 74, 18 (25). 1916. $\left.-{ }^{39}\right)$ v. Tabora, Nr. 4, S. 377. 